\title{
Improving asthma severity and control screening in a primary care pediatric practice
}

\author{
Sathyanarayan Sudhanthar, Jane Turner, Yakov Sigal, Kripa Thakur, Jonathan Gold \\ Pediatrics and Human Development, CHM, Michigan State University
}

\begin{abstract}
Asthma is the most commonly encountered chronic disease in children. Periodic assessment of asthma severity and control is an integral part of asthma management, but patients with uncontrolled asthma don't always schedule routine asthma care visits. The aim of this project was to improve asthma control and severity screening in a primary care setting by using a validated tool for all visits for patients with a diagnosis of asthma aged 4-21 years. Our QI team developed a protocol to administer the Asthma Control Test (TM), a validated questionnaire to assess asthma control. The stakeholders involved were the physicians, nursing staff, and the Health Information Team (HIT). All patients who had a prior diagnosis of asthma or with an asthma medication in their chart, who presented for any clinical visit including asthma were administered ACT. The staff scored the ACT and included the form in the encounter sheet so that the physicians can review the scores, address the asthma control, severity, and document in the chart. The number of patients whose asthma control was assessed improved from $10 \%$ per year to $85 \%$ after the three PDSA cycles. Administration of the tool did not impact the flow of the patients in a busy primary care practice. Screening asthma severity and control for patients diagnosed with asthma with a validated questionnaire when presenting for any chief complaint including asthma will help the provider address the severity and control of asthma symptoms in a timely manner and would potentially help prevent unwanted emergency department or urgent care usage.
\end{abstract}

\section{Problem}

The Pediatric primary care practice in Michigan State University is an academic practice with eight physicians serving about 5500 children in the community. Among our clinic population, a significant portion of them have Medicaid insurance (56\%). The clinics also serve as a teaching clinic to third and fourth year medical students and residents during their pediatric rotations. Internal review among the staff and the providers yielded a great variability in assessing severity of asthma symptoms in our patient population. It was also noted that patients with asthma who presented for visits such as sports physicals, evaluation, and follow up of mental health problems, development, and behavioral problems were not being assessed routinely about the severity and control of their asthma. In many instances children with asthma, end up in the urgent care or emergency. This is a problem because it is a waste of health care resources and not a good example of coordinated care that as a practice we strive to achieve. It is also a poor example for Medical Home model which strives to provide a comprehensive, patient centered, coordinated, accessible, and a competent care.[1] The aim of this project was to improve the asthma control and severity screening by $80 \%$ within the next 12 months, by developing a clinic flow protocol using the validated tool to be administered at all types of visits and to all the patients with a diagnosis of asthma ages 4-21 years.

\section{Background}

Asthma is one of the most common chronic diseases in United States. Uncontrolled asthma is responsible for a significant amount of lost school days for children and work days for parents .
Assessing control during primary care visits for reasons other than asthma is a potential strategy to address this gap. The 2007 National Heart Lung Blood Institute (NHLBI) guidelines clearly state the importance of assessing asthma severity and control during each visit. The lifetime prevalence of asthma is $12.7 \%$ nationally[2] and more than half $(57 \%)$ of children diagnosed as having asthma will end up having a severe asthma exacerbation[3]. A majority $(60 \%)$ have persistent symptoms and among this category about $38 \%$ have uncontrolled asthma[4]. Poor control of asthma usually leads to missed school days for the child and work days for the parents[5]. The number of missed school days among children with asthma in 2013 is estimated to be 13.8 million and about half the children in the age groups of 5-17 years of age who have been previously diagnosed with asthma said they missed school because of poor asthma control (6). The National Asthma Education and Prevention Program Expert Panel -3 2007, recommends assessing asthma control and severity as a focus for adjusting the therapy in patients diagnosed with asthma. Asthma control can be assessed by a validated tool such as Asthma Control Test (TM (ACT)[7]. This is a validated tool developed by Glaxo-Smith Kline and used to assess the control of the asthma symptoms in patients and a score of 19 or less is considered as poorly controlled asthma[8]. ACT has also been validated for use by mail and over phone to assess the asthma control of a patient.[9,10]

\section{Baseline measurement}

For this project, we collected baseline measurement retrospectively by reviewing clinical encounters of 100 randomly selected patients with an International Disease Classification-9 (ICD-9) diagnoses of asthma, wheezing, or reactive airway disease who had a clinical encounter in the 12 months prior to implementation of the asthma 
assessment tool specifically from March 2012 - February 2013. The clinical encounter could be for an asthma exacerbation, annual health maintenance, behavioral and developmental disorders, or just a sick visit unrelated to asthma. Patients who primarily had asthma related visits were administered the ACT tool more frequently than for any other visits. Among the 100 patients reviewed, only 12 patient charts (12\%) had documentation of ACT being administered, scored, and a change in management was performed depending on the score. All of those 12 patients specifically had presented for an asthma flare. This represents a misunderstanding on the part of the provider as the ACT is more helpful in assessing the severity and control of asthma, when patients are not having active asthma symptoms.

\section{Design}

It was clear that the practice had to make a decision of using a quick and reliable validated tool to assess asthma control and severity. Most of the practitioners were amenable to the change though the time factor to administer the questionnaire and score them was a major concern. A QI team consisting of important stakeholders including a physician champion, nurse champion, representative from HIT, and other staff members were formed. A clinic flow protocol was developed with the involvement of the key stakeholders. As per the protocol, when the patients were roomed in, the nursing staff checked the problem list for a diagnosis of asthma, reactive airway disease, or wheezing, etc. They also checked the medication list for usage of rescue medication such as Albuterol, inhaled steroids, or leukotriene Receptor Antagonist. If they identified the patient as having either one or both of the criteria, then the staff printed out the ACT which is incorporated within the Electronic Medical Records (EMR), and gave it to the patients or the family. Once the form was completed the nursing staff scored and included with the encounter sheet for the physicians to look at it. If the score was 19 or more the asthma control was optimal. If the score was less than 19 providers addressed the asthma control with the patients.

The implementation team met about every four weeks during the cycles to monitor the implementation of the ACT. Also, the providers and staff were educated about the tool, its validity and questions about the clinical flow were answered. New providers and staff got orientation from nurse and physician champions and were also included in the monthly meetings.

\section{Strategy}

In our first PDSA cycle we decided to implement the ACT to children with a diagnosis of asthma from ages 12-21 who presented for any visit including asthma. This was done to find out the impact of filling out the validated questionnaire during busy clinic hours. The 12 year old and above form is filled by the patient primarily and hence the stakeholders decided that would be the first step. After a few discussions with the HIT the ACT form was incorporated in the EMR. Also, Glaxo-Smith Kline, the company that holds the license to this form was also contacted and permission was granted to use the ACT for our clinic patients. This PDSA cycle was primarily done to test the feasibility of administering the ACT forms to the asthma patients irrespective of the type of visit. The informal feedback from the staff, providers, and patients were mostly positive and helped us to go through the second cycle.

In our second PDSA cycle, we implemented the ACT to children with a diagnosis of asthma from the age of 4-11 years who presented for any visits including asthma. The 4-11 age, asthma questions were filled out by both the parent and the patient. The nursing staff continued to score and attach the form to the billing sheet so that the providers can view them. Since the goal was to incorporate this tool more broadly, the aim of the second cycle was to test the feasibility of administering the ACT to all children from ages 4-21 presenting for any visit to assess their asthma severity and control. Feedback from patient and providers were positive. The staff did give feedback about the impact on their time but were still willing to administer the questionnaire as it was perceived to be a helpful clinical tool.

In our third PDSA cycle, we continued incorporating the asthma form for all the patients who were diagnosed with asthma from ages 4-21 years to be given at all the visits they present for, irrespective of if they were presenting with asthma related symptoms or not. We also developed a short text for the providers to document the findings of the ACT in the EMR. Continued education of new providers and staff members were carried out throughout the cycle and up to six months after the completion of PDSA 3.

\section{Results}

We had our post intervention measurements at the end of each PDSA cycles as well as three months and six months after the end of PDSA cycle 3 . The asthma severity assessment using a validated tool like the Asthma Control Test improved from $10 \%$ to $50 \%$ three months post PDSA cycle 3 , and $85 \%$ six months post PDSA cycle 3 . The ACT also gave opportunity for the providers to review the Asthma Action Plans with their patients, refill necessary medications, address adherence, and correct techniques for inhaler and spacer use.

See supplementary file: ds6981.pptx - "Run chart of percentage of asthma patients screened with ACT."

\section{Lessons and limitations}

There were a few lessons we learnt from this project. Anecdotal collection of information from staff and parent - patient interaction denotes about two minutes to fill the form and 30 seconds to score the form. The providers agreed that the time spent was reasonable as they were able to address poorly controlled asthma, initiate or modify treatments, check for their Pulmonary Function Test, and update their Asthma Action Plans. It was also noted because of more complexity in the visit providers usually billed a higher level of service and that would compensate for the extra time spent by the clinic staff in assessing the severity of the asthma symptoms. Also, many providers missed documenting the asthma severity assessment in the EMR and hence having them scan the ACT form 
in the EMR provided the baseline score on the asthma control which could also be referred back and compared in the follow-up visits. Even though we did not calculate the dollar amount saved by assessing severity and control of asthma in visits other than the scheduled asthma visits by preventing potential emergency or urgent care visits, guidelines suggest continued monitoring of asthma severity is an important step to save valuable health care expense.

\section{Conclusion}

Instituting an universal way of assessing the asthma severity and control to all the patients at all visits with an asthma diagnosis with a quick, reliable, and validated tool as a workflow process was successful. At the time of this submission, though there were many studies documenting the reliability and validity of the ACT, we found no major studies in PubMed detailing the implementation of the ACT tool for all visits in a primary care practice. Using a validated tool such as ACT, takes the guesswork out of how well the patients are coping with their asthma symptoms and gives an opportunity for reviewing patient adherence, action plan, and education. The ACT tool administration could be easily replicated in other primary care practices as it had very minimal impact on flow of our busy clinics. The information from such a validated tool could also be a focus of discussion with patients and parents about their child's asthma control. Administration of this tool is also sustainable as all our pediatric practices have continued to administer the tool for the last 18 months, well after the end of the QI project. The next step for the QI team will be to assess if primary care screening for asthma control at all visits with a validated tool prevents emergency department visits for our patients with persistent asthma and possibly help decrease valuable health care dollars spent due to such visits.

\section{References}

1 Patient-Centered Primary Care Collaborative [Internet]. Accessed on 11/17/15. Available from: https://www.pcpcc.org/about/medicalhome

2 Centers for Disease Control and Prevention [Internet]. Accessed on $11 / 17 / 15$. Available from:

http://www.cdc.gov/asthma/nhis/2013/table2-1.htm

3 Centers for Disease Control and Prevention [Internet]. Accessed on $11 / 17 / 15$. Available from:

http://www.cdc.gov/asthma/nhis/2013/table6-1.htm

4 Centers for Disease Control and Prevention [Internet]. Accessed on $11 / 17 / 15$. Available from:

http://www.cdc.gov/asthma/asthma stats/severity child.htm

5 National Heart, Lung, and Blood Institute. Expert Panel Report 3: Guidelines for the Diagnosis and Management of Asthma.

Bethesda, MD: National Heart, Lung, and Blood Institute; 2007.

6 Centers for Disease Control and Prevention [Internet]. Accessed on $11 / 17 / 15$. Available from: http://www.cdc.gov/asthma/asthma_stats/default.htm

7 Nathan RA, Sorkness CA, Kosinski M, et al. Development of the asthma control test: A survey for assessing asthma control. J Allergy Clin Immunol 2004 (113): 59-65

8 Schatz M, Sorkness CA, Li JT, et al. Asthma Control Test: reliability, validity, and responsiveness in patients not previously followed by asthma specialists. J Allergy Clin Immunol. 2006 Mar;117(3):549-56.

9 Schatz M, Mosen DM, Kosinski M, et al. Validity of the Asthma Control Test completed at home. Am J Manag Care 2007;13:661-7.

10 Schatz M, Zeiger R, Drane A, et al. Reliability and predictive validity of the Asthma Control Test administered by telephone calls using speech recognition technology. J Allergy Clin Immunol 2007;119: 336-43.

\section{Declaration of interests}

None declared

\section{Acknowledgements}

MSU-CHM Primary care Pediatric team including Drs.Shiwaji Pawar, Jessica Gengler, Mike Stiffler, Nursing staff, and Health Information Team.

\section{Ethical approval}

Michigan State University Institutional Review Board considers this project as a Quality Improvement project only and does not mandate going through the IRB. 